E-mail: darja.frankic@lek-zbor.si

\section{Supply of Medicinal Products in the Hospital - How Long Can We Hold Out?}

Tajda Mibarija-Gala; Andreja Vidmar; Silva Jenko Pharmacy, University Medical Centre, Ljubljana, Republic of Slovenia

At the University Medical Centre (UMC), medicinal products (MP) and medical devices (MD) are stored at five locations: (1) the pharmacy, (2) clinical departments with their own stock; (3) MD warehouse; (4) national reserves of MP, kept in the pharmacy; and (5) stock of the Defence, Security, and Safety Service in the UMC. The UMC pharmacy has approximately 1,400 MP available in different pharmaceutical forms and substances for the preparation of galenic and "ex tempore" preparations. The supplies of MP are limited and can cover a period of up to 14 days, while the stock of certain imported drugs (such as antidotes and MP used in transplantations) can cover a longer period of time.

In the UMC wards, regular audits are conducted of consumption and storage of drugs and to maintain the cold chain of the vaccine supply. In the UMC pharmacy, the national reserves of MP are stored and updated. The quantity of the reserves is adjusted to a level sufficient to cover three months of consumption by the UMC. However, we are aware of the increased requirements for specific MP that must be met during emergency situations. Good communication, a well-organized transport service, and a high level of awareness among the pharmacy staff members are of key importance for an appropriate supply of MP, and their rapid and efficient distribution to the injured in an emergency situation.

Keywords: audit; consumption; disaster; emergency; pharmacy; requirements; storage; stores; supplies; transport

Prebosp Disast Med 2003:18:s(1)s8.

E-mail: tajda.gala@kclj.si

\section{Preparation of Parenteral Solutions -}

\section{How Far We May Deviate From the Regulations?}

Tajda Mibarija-Gala; Valda Vodopivec

Pharmacy, University Medical Centre, Ljubljana, Republic of Slovenia

Parenteral therapy is fundamental in the hospital environment, as it allows for rapid introduction of drugs into the body and facilitates a rapid onset of its action. The primary raw materials for the preparation of parenteral products are purified water and water for injections. They are prepared from microbiologically pure drinking water using a special procedure that involves considerable energy consumption. The manufacture of sterile products must be carried out in clean areas, i.e., following extremely stringent preparation requirements to minimize the risk of contamination with micro-organisms, mechanical particles, and pyrogens. Sterile solutions are prepared using sophisticated equipment that is regularly maintained, serviced, and validated. The whole process of preparing these products is supported by appropriate production documentation. All of the products are subjected to quality control and analyses in line with the current regulations.

The proper conduct of the production process requires highly technically qualified pharmaceutical personnel with thorough knowledge of the principles of quality work in aseptic environments. As a rule, in emergency situations an increased need for sterile solutions occurs, which, considering the small scale of production in a hospital pharmacy setting, greatly affects the dynamics of the process of preparation of the solutions. In view of the fact that the regular market supply of solutions will be hindered or even prevented in emergency situations, it is even more important to preserve the operation of this type of manufacturing facilities.

Keywords: disaster; emergency; manufacture; personnel; preparation; process; production; solutions, parenteral; supplies

Prehosp Disast Med 2003:18:s(1)s8.

E-mail: tajda.gala@kclj.si

\section{Importance of Aseptic Preparation of Products for External Use \\ Tajda Mibarija-Gala; Simona Mitrovic; Spela Urb \\ Pharmacy, University Medical Centre, Ljubljana, Slovenia}

Good manufacturing practice requires that some medicinal products for external use should be prepared with aseptic techniques. These include: (1) eye preparations; (2) solutions for inhalation; (3) disinfectants; (4) products containing antimicrobials; (5) agents for stimulation of epithelization; and (5) ointments for the treatment of burns. Essential conditions: The area, personnel, equipment, and documentation have to be in line with the current regulations.

For the treatment of injuries and eye diseases and for diagnostic and test purposes, we prepare a broad range of eye drops and eye irrigation solutions in water or in oil and ointments. For ophthalmic surgical procedures, we prepare methyl-cellulose gels in intraocular solution. For the treatment of chronic diseases and for patients in intensive care units, we prepare inhalation solutions that may contain various active substances.

The pharmacy prepares aseptically disinfectants for skin and mucous membranes. Appropriate disinfection is the basic principle of the prevention of hospital infections; the whole system is led and supervised by the Commission for the Prevention of Hospital Infections.

In emergency situations, disinfectants are of special importance, as the risk of spreading of micro-organisms and epidemic outbreaks is greatly increased. In emergency situations such as war, catastrophes, or natural disasters, an uninterrupted supply of aseptically prepared products must be ensured that considers both the nature of the problems posed by the given situation and the requirements to be met in aseptic preparation.

Keywords: antimicrobial; catastrophes; disasters; disinfectants; epidemics; infections; intensive care; intraocular; nebulization; pharmacy; preparations; topicals

Prebosp Disast Med 2003:18:s(1)s8.

E-mail: tajda.gala@kclj.si 